\title{
Spatial memory decline after masticatory deprivation and aging is associated with altered laminar distribution of CA1 astrocytes
}

\author{
Marina Negrão Frota de Almeida', Fabíola de Carvalho Chaves de Siqueira Mendes', \\ André Pinheiro Gurgel Felício ${ }^{1}$, Manoela Falsoni ${ }^{1}$, Márcia Lorena Ferreira de Andrade ${ }^{1}$, João Bento-Torres ${ }^{1}$, \\ Pedro Fernando da Costa Vasconcelos ${ }^{2}$, Victor Hugh Perry ${ }^{3}$, Cristovam Wanderley Picanço-Diniz ${ }^{1}$ and \\ Marcia Consentino Kronka Sosthenes ${ }^{1,4^{*}}$
}

\begin{abstract}
Background: Chewing imbalances are associated with neurodegeneration and are risk factors for senile dementia in humans and memory deficits in experimental animals. We investigated the impact of long-term reduced mastication on spatial memory in young, mature and aged female albino Swiss mice by stereological analysis of the laminar distribution of CA1 astrocytes. A soft diet (SD) was used to reduce mastication in the experimental group, whereas the control group was fed a hard diet (HD). Assays were performed in 3-, 6- and 18-month-old SD and HD mice.

Results: Eating a SD variably affected the number of astrocytes in the CA1 hippocampal field, and SD mice performed worse on water maze memory tests than HD mice. Three-month-old mice in both groups could remember/find a hidden platform in the water maze. However, 6-month-old SD mice, but not HD mice, exhibited significant spatial memory dysfunction. Both SD and HD 18-month-old mice showed spatial memory decline. Older SD mice had astrocyte hyperplasia in the strata pyramidale and oriens compared to 6-month-old mice. Aging induced astrocyte hypoplasia at 18 months in the lacunosum-moleculare layer of HD mice.

Conclusions: Taken together, these results suggest that the impaired spatial learning and memory induced by masticatory deprivation and aging may be associated with altered astrocyte laminar distribution and number in the CA1 hippocampal field. The underlying molecular mechanisms are unknown and merit further investigation.
\end{abstract}

\section{Background}

Previous studies have established an association between chewing activity and cognition [1-3]. The systemic effects of long-term masticatory imbalances are associated with neurodegeneration and are a risk factor for senile dementia in humans [4] and memory deficits in experimental animals [5]. To investigate the impact of masticatory imbalances on various activities and physiological factors, experimental masticatory deprivation has been modelled experimentally in animals using a modified diet [6], molar removal $[7,8]$, or occlusion disharmony modelled

\footnotetext{
* Correspondence: makronka@gmail.com

'Universidade Federal do Pará-UFPA, Instituto de Ciências Biológicas, Laboratório de Investigações em Neurodegeneração e Infecção, Hospital Universitário João de Barros Barreto, Belém, PA, Brazil

Full list of author information is available at the end of the article
}

by "bite-raised" condition $[9,10]$. These approaches revealed that masticatory dysfunction reduces spatial learning and memory in water maze tests in rats $[8,11]$ and mice [11-13], and that these deficits seem to increase with aging and time after tooth loss [8]. Induced molarless subjects, revealed reduced neurogenesis in young rats [14], and there is a loss of astrocytes, neurons and dendritic spines in the hippocampus of aged rats and mice $[7,14,15]$. Young mice fed a soft diet show reduced neurogenesis and BDNF levels $[16,17]$, whereas progressive synaptic reduction and pyramidal neuron losses are observed in aged mice [6,18].

Masticatory deprivation seems to affect mainly the hippocampus of all ages, and young, middle-aged and senile mice subjected to masticatory imbalances show a

\section{() Biomed Central}


decreased number of neurons in CA1 $[11,18]$ and CA3 [18] and an increased number of hypertrophic astrocytes in CA1 [8]. All of these changes seem to be aggravated by aging [15] and time after tooth loss [11], suggesting additive effects. However, to our knowledge, no stereological analysis has been conducted to date that explores the long-term glial changes induced by a soft diet regime imposed early in life.

Notably, cerebral blood flow in various areas of the brain is affected by mastication [19-21], and astrocytes are likely to play a key role in regulating cerebral blood flow [22-25]. In particular, the regulation of blood flow seem to be mediated by astrocyte calcium $\left(\mathrm{Ca}^{2+}\right)$ signalling, which may induce both arteriolar dilation and constriction as a function of calcium concentration [25].

Since previous results demonstrated that aging affects spatial memory and astrocytes in the dentate gyrus [26] and hippocampus [27] in a laminar-dependent fashion, we tested the hypothesis that long-term masticatory deprivation and aging have additive effects on spatial memory and on the laminar distribution of CA1 astrocytes as assessed by an optical fractionator, which is an unbiased stereological analysis method.

\section{Results}

Masticatory reduction and water maze tests in 3-, 6- and 18- month-old mice

Figure 1 shows a graphic representation of the escape latencies for water maze tests conducted on five consecutive days on mice that were 3-, 6- and 18-months old. Three-month-old SD and HD mice showed similar performance on the water maze test. Indeed, both groups showed significant decreases in the escape latencies on the 3 rd day test as compared to the 1st day test (Figure 1). For the 6-month-old mouse groups, only the HD mice were able to significantly reduce latency values over the 5-day period. Finally, 18-month-old mice from both the SD and HD groups were unable to reduce escape latency during the 5-day testing period, and there were no significant differences in the escape latencies on the 1st and the 5th test days in either group. Analysis of the swim paths revealed that the SD group had, overall, longer and more erratic trajectories compared to age-matched HD mice (Figure 1, inset). Neither masticatory deprivation nor aging had a significant influence on swimming speed, suggesting that the negative effects on latency were due to impaired spatial memory rather than to alter swimming per se.

Figure 2 shows photomicrographs of CA1 from the indicated mouse groups as well as the laminar distribution of astrocytes; graphs of the mean number and standard errors for the estimated number of astrocytes in the strata lacunosum-moleculare, radiatum, pyramidale and oriens are also shown. (For detailed data, see Additional file 1: Table S1, Additional file 2: Table S2, Additional file 3:

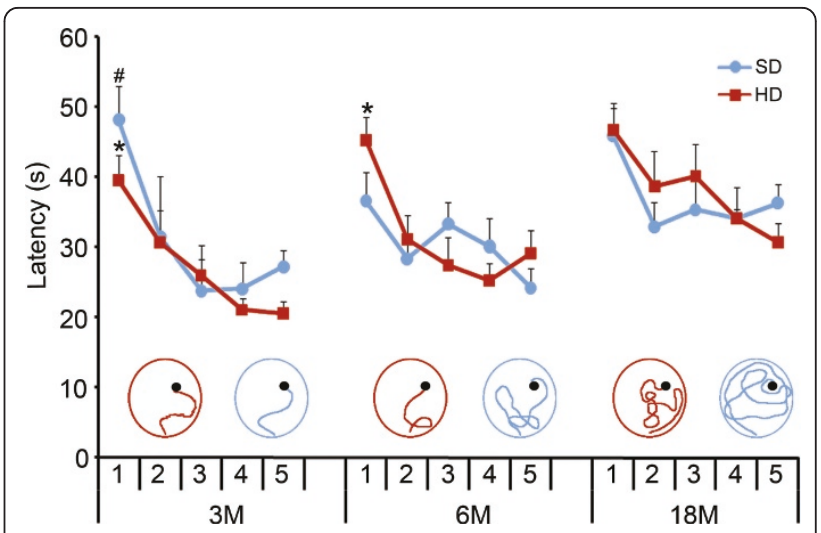

Figure 1 Performances on the water maze tests applied to 3-, 6- and 18-month old mice were compared by determining the escape latencies on five test days. Three-month-old SD and HD mice showed similar latencies, while the escape latencies of 6month-old HD mice were significantly shorter than those of SD agematched mice. SD and HD 18-month-old mice did not show any significant change in escape latencies. Swimming tracking analysis revealed that SD had longer trajectories as compared to agematched HD mice, particularly at 6 months of age (inset). $\mathrm{HD}=$ hard diet; $\mathrm{SD}=$ soft diet $\left(^{*}=p<0.05\right.$ for the HD group. $\#=p<$ 0.05 for the SD group)

Table S3 and Additional file 4: Table S4, Additional file 5: Table S5, Additional file 6: Table S6, Additional file 7: Table S7 and Additional file 8: Table S8). The representative sections chosen for Figure 2 were from mice that had an estimated number of astrocytes that was close to the mean group value. It is clear in the photomicrographs that there are qualitative age-related changes in the control "normal diet" HD group. From 3 months to 6 months, there was a marked increase in branching density associated with changes in the number and intensity of glial fibrillary acidic protein (GFAP) immunolabeled branches. There was strong GFAP immunolabeling near the arteries in the hippocampal fissure, suggesting a role for astrocytes in local blood flow in these layers of CA1. However, by 18 months there was a clear reduction in branching density and marked atrophy of the branching as revealed by GFAP immunolabeling. Changes in astrocyte number were quantified, revealing that there were significantly fewer astrocytes in 3-month-old SD mice compared to 3-monthold HD mice in all CA1 layers (two-tail $t$-test, $p<0.05$ ). However, 6-month-old mice only showed SD vs. HD differences in the stratum pyramidale (two-way ANOVA, Bonferroni post-hoc tests, $p<0.05$ ). In 18 -month-old mice, there was an increase in the number of astrocytes in the strata lacunosum-molecular and oriens of HD mice compared to SD mice (two-way ANOVA, Bonferroni post-hoc tests, $p<0.05)$. Aging-induced astrocytosis in all CA1 layers was evident in 18-month-old SD mice compared with 3-month-old mice. However, the only significant differences noted in 6-month-old SD mice compared with 


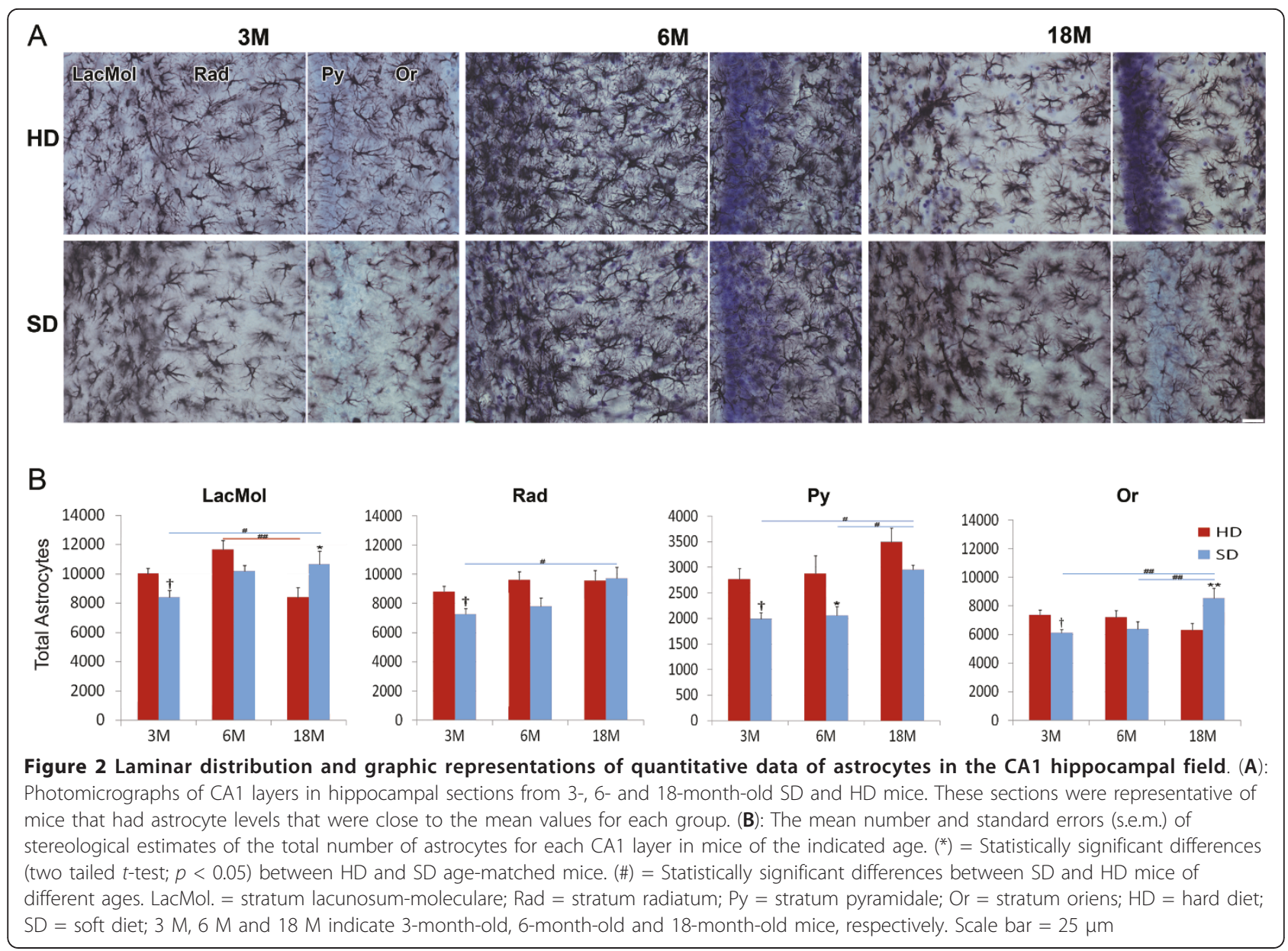

18-month-old SD mice were in the strata pyramidale and oriens. Thus, aging and diet seemed to induce additive astrocytosis, particularly in the strata lacunosum moleculare and oriens (two-way ANOVA, $p<0.01$ ). Overall, the main effects of the SD were to reduce the number of astrocytes at 3 and 6 months and then to prevent further agerelated losses at 18 months in the lacunosum-moleculare layer.

The HD and SD groups showed significant differences in the total number of astrocytes in CA1 at 3 months but not at 6 months or 18 months. Indeed, SD mice had, on average, fewer astrocytes at 3 months $(23,839 \pm 653)$ compared to age-matched HD mice $(28,999 \pm 807),(p=0.002)$. However, there were no differences in the total number of astrocytes in SD vs. HD mice at 6 months $(26,477 \pm 1572$ vs. $31,440 \pm 1613)$ or 18 months (31901 \pm 2046 vs. 27780 $\pm 1965)$, $(p>0.05)$, respectively. Aging only induced significant astrocytosis in SD mice at 18 months compared with SD mice at 3 months and 6 months.

\section{Discussion}

The results of the present study demonstrated that 24hour-per-day masticatory deprivation had differential laminar effects on the number of astrocytes in CA1 and on water maze memory test performance in 3-, 6- and 18-month-old female albino Swiss mice. We suggest that masticatory deprivation can induce abnormal cognitive development and may enhance changes in aging astrocytes in CA1.

\section{Hippocampal astrocytes, water maze performances and reduced mastication}

In the present report, water maze tests revealed that after 6 or 18 months of masticatory deprivation, experimental SD mice could not learn and remember the position of a hidden platform; in contrast, control 6-month old HD mice could learn and remember the position of a hidden platform, whereas 18-month-old mice from both groups $\mathrm{SD}$ and $\mathrm{HD}$ were unable to reduce escape latency during the 5-day testing period. There were no significant differences in the swimming speeds of young (3-month-old), mature (6-month-old) and aged (18-month-old) mice in either the SD or the HD group, suggesting that spatial memory impairment, rather than motivation or physical performance, was a more likely explanation of the differences in the water maze performance. In parallel with 
memory dysfunction, there were significant changes in the laminar distribution of astrocytes in CA1. A continuous SD that started early in life $\left(21^{\text {st }}\right.$ postnatal day) induced astrocytic hypoplasia at 3 months in all layers and in the pyramidal layer at 6 months as compared to age-matched HD mice. Compared to 18-month-old SD mice and 6-month-old HD mice, 18-month-old HD mice showed hypoplasia in the strata lacunosum moleculare. In addition, 18-month-old SD mice showed significantly more astrocytosis than 3-month-old and 6-month-old SD mice. Taken together, the results suggest that masticatory deprivation induced abnormal cognitive development and may have enhanced changes in aging astrocytes in CA1.

Although there were no learning and memory differences in the water maze test [28] after 3 months of masticatory deprivation, the laminar distribution of CA1 astrocytes started to change when the SD mice were 3 months old and became especially severe after six months or more of masticatory deprivation. Since spatial memory and learning were investigated previously only in adult (6-month-old) but not in aged (18-month-old) albino Swiss mice [29-31], this is the first report to assess the effects of long-term masticatory deprivation (using SD to model deprivation) on astrocytes by stereological analysis of CA1.

The accelerated cognitive decline observed in SD mice was not directly correlated with time-specific changes in astrocyte number in any CA1 layer. Thus, we investigated whether there were morphological changes (e.g. evident shrinkage of astrocyte arbors) that affected the astrocyte network. Long-term potentiation (LTP), considered the neurophysiological basis for learning and memory, is facilitated by brain-derived neurotrophic factor (BDNF) in both young and aged mice. However, in aged mice the effects of exogenous BDNF on LTP does not translate into improved spatial memory [32]. Interestingly, BDNF expression in the hippocampus of $\mathrm{C} 57 \mathrm{Bl} 6$ mice is reduced under SD as compared to $\mathrm{HD}$ at 3 and 6 months [16]; in parallel, there is reduced neurogenesis in SD mice of the same ages [17]. We also observed a reduction in the number of astrocytes at 3 and 6 months in SD mice vs. HD mice. Notably, astrocytes synthesize and release BDNF, which is critical for experience-dependent synaptic plasticity in the mature brain [33], and there is a reduction of hippocampal BDNF levels in aged mice, with deleterious consequences for synaptic plasticity and spatial memory [34].

In the present report, there were significantly more astrocytes in the stratum oriens at 18 months in SD mice compared to HD mice. The combination of aging and masticatory deprivation affects the stratum oriens in an additive manner, reducing both dendritic spine density [7] and synaptic density [6] and significantly reducing cell size in SD and HD groups. These results suggest that astrocytic changes induced by aging and masticatory deprivation are greater in the strata lacunosum-moleculare and radiatum than changes in the strata pyramidale and oriens.

Many studies show that the CA1 is important in signalling pathways that are key for learning and memory (reviewed in [35]). Specifically, the lacunosum-moleculare layer is the target of the temporoammonic pathway, which originates in the entorhinal cortex and creates excitatory glutamatergic synapses with distal pyramidal dendrites in CA1 in mice [36] and rats [37,38]. Temporoammonic synapses are associated with theta oscillations and late-phase LTP and long-term memory consolidation [39-41]. In addition, astrocyte calcium signals at Schaffer collateral to CA1 pyramidal cell synapses correlate with the number of activated synapses [42], demonstrating the direct participation of astrocytes in the hippocampal circuits that are involved in spatial memory. The presence of synaptic potentiation has been described previously to occur via astrocytic glutamate exocytosis at the entorhinal-to-dentate granular cells (the perforant pathway) $[43,44]$ and Schaffer collaterals [45]. In this way, astrocytes play a critical role in memory functioning and LTP and contribute to synaptic tuning in the hippocampus [44].

Astrocytes in the hippocampus seem particularly sensitive to age-related changes [46-48] and can be impaired by structural/functional changes induced by masticatory imbalances (see, for example, [5-7]). Indeed, impairment of spatial memory is reported to occur in aged mice when the molar teeth are extracted or cut when the mice are young [11] as well as in adult rats that are fed a SD after the weaning period [6]. In these studies, both neuronal density in the CA1 hippocampus [11] and synaptic formation in the hippocampus and the parietal cortex [6] were reported to decrease. In agreement with these studies, tooth loss and the resulting masticatory alterations leads to a reduction in the number of ChAT-positive neurons in the Broca diagonal band and medial septum, resulting in a decrease of acetylcholine levels in the hippocampus $[49,50]$.

Most of the earlier studies of the impact of reduced mastication on the hippocampus focused on neuronal changes. To our knowledge, there are only a few reports that employed GFAP-immunolabeling of the CA1 hippocampal field, and all such studies were restricted to aged SAMP8 mice. These studies revealed that the molarless condition enhances the age-dependent increase in the density and hypertrophy of astrocytes, and that these effects increase the longer the molarless condition persists $[8,15]$.

The notion that aging and masticatory deprivation may cause similar cellular and molecular alterations in the hippocampus is supported by other studies (reviewed in 
[51]). Indeed astrocyte hypertrophy has been reported previously in the CA1 hippocampal field $[8,15]$, suggesting that these cells may increase their production of proinflammatory cytokines in response to inflammation. Similar changes have been described in the senile hippocampus, where a more reactive astrocyte phenotype is expressed during aging, even in the absence of neurological disease, as part of an increased and maintained proinflammatory profile that may be associated with cognitive dysfunction (reviewed in [52]).

It is unclear why the number of astrocytes in the stratum lacunosum-moleculare was reduced in 18-month-old HD mice compared to 6-month-old HD mice. However, since BDNF regulates gliogenesis, it could be that the reduced levels of BDNF induced by aging contributed to the decrease in the number of neurotrophic astrocytes [53]. In addition, we suggest that the astrocytosis observed in the stratum oriens of 18-month-old SD mice is not dependent on BDNF but, rather, is part of the inflammatory aging process [52]. Although the present investigation is related to chronic stress (since there was a long period of masticatory deprivation), previous results regarding the acute stress response during immobilization revealed that biting can restore the BDNF mRNA levels that are reduced by stress [54]. Taken together, the results suggest that a decrease in masticatory activity along with aging may promote the observed differential laminar effects on CA1 astrocytic plasticity.

\section{Astrocytes, aging and CA1 blood flow changes}

The strong GFAP immunolabeling we observed near the arteries in the hippocampal fissure suggests a role for astrocytes in local blood flow regulation in these layers of the CA1 and dentate gyrus. Indeed, there is some evidence that astrocytes are involved in hippocampal neurovascular control [24]. Astrocytes contribute to local blood flow in the hippocampus, and we observed a statistically significant reduction in the number of GFAP-immunolabeled cells in the lacunosum moleculare of CA1 in HD mice late in life; thus, we predict that hippocampal blood flow is altered in 18-month-old mice. The decrease in GFAP immunolabeling may be secondary to a reduction of neuronal activity induced by aging in the temporoammonic pathway in mice $[36,55]$. However, since the astrocytic atrophy in SD mice seemed more severe than in HD mice, an alternative interpretation is that aging and a SD could be related to the decrease in functional memory at 18 months. Since we did not investigate possible correlations between the density of GFAP-positive processes and astrocyte function, this remains a key issue that merits further investigation. Similar alterations occur in the human hippocampus [56], and a major disturbance in cerebral blood flow late in life that is associated with axon-glial disruption could link vascular disease and chronic neurodegenerative diseases that are associated with aging [57]. Since tooth loss [58] and neurovascular dysfunction [59] seem to predict poor cognitive function in older humans, it is reasonable to hypothesize that masticatory deprivation in humans contributes, at least in part, to spatial memory dysfunction in aging [60]. Changes in hippocampal astrocytes may be associated with this as well.

\section{Astrocytic changes, corticosteroids and masticatory stress}

Active mastication can rescue the stress-attenuated hippocampal memory process in animals by suppressing endocrinological and autonomic stress responses [51]. Indeed, previous studies of molarless mice showed plasma corticosterone levels that were significantly greater than those in molar-intact control mice. In addition, pretreatment with suppressors of stress-induced increases in plasma corticosterone levels prevented the molarless-induced increase in plasma corticosterone levels [61]. In addition, elevated corticosterone levels suppress synaptic plasticity in the hippocampus [62], and mastication suppresses the stress-activated expression of corticotropin-releasing factor (CRF)[63]. Astrocytic plasticity in the hippocampus may also be affected by corticosteroids due to changes in the number of astrocytes $[64,65]$ or to changes in astrocyte morphology and function [66]. In particular, corticosterone increases the number of astrocytes in CA1 in a dosedependent fashion [64].

In this study, masticatory deprivation-induced stress may have altered the plasma glucocorticoid levels, and this may in turn have affected astrocytic plasticity. We did not measure plasma corticosteroid levels; therefore, we cannot exclude the possibility that altered levels of corticosteroids might explain the observed effects of masticatory stress. However, there is currently no information about changes in glucocorticoid levels that might be induced by a SD, so it is difficult to evaluate this possibility.

\section{Hormones and astrocytes}

It has now evident that ovarian steroids influence cognition and aging $[67,68]$ and that hormonal cycle disruption late in life is associated with memory impairment $[69,70]$. In addition, females and males show striking differences in cognitive decline associated with aging, mainly in visuospatial abilities [71]. Because females are more susceptible to the cognitive decline that accompanies aging, it would be expected that the additive effects of estropause and aging would be greater than in males. Interestingly, aged C57Bl6J female mice have $18.3 \%$ more astrocytes than young female mice and $32 \%$ more than aged male mice [72]. Since the SD and HD mice in the present report were age-matched, it is reasonable to assume that mice in both 18-month-old groups were in estropause and to hypothesize that the significant differences in spatial memory and CA1 astrocytes detected in 
these mice were due to differences in diet as well as to aging.

\section{Non-stereological technical limitations}

Estimations of the number of objects of interest in stereological studies can vary among studies. To detect the effects of variations in the criteria for identifying objects of interest in this study, we looked at the polymorphic layer of the dentate gyrus in aged female albino Swiss mice, which was previously described as having an increased number of astrocytes (aged $=10,232 \pm 1325$ vs. young $=6440 \pm 1085, p<0.05)$ [73]. As expected, we found that there were more astrocytes in the polymorphic layer of aged HD mice (18-month-old HD = $11,101 \pm 1904)$ compared to young mice (6-month-old $\mathrm{HD}=8162 \pm 1354 ; p<0.05)$.

The level of acceptable errors for stereological estimations was defined as the ratio between the intrinsic error introduced by the methodology and the coefficient of variation $[74,75]$. The coefficient of error (CE) reflects the accuracy of the cell number estimates, and CE values $\leq$ 0.05 were deemed appropriate for the present study since the variance introduced by the estimation procedure contributed little to the observed group variance. As a result, variations associated with non-biological sources were reduced to acceptable levels in the present report $[72,75]$.

\section{Conclusions}

Taken together, the results of this study suggest that the abnormal spatial learning and memory induced by longterm masticatory deprivation and aging may be associated with altered laminar distribution and a reduced number of astrocytes in CA1, as demonstrated by stereological analysis. The molecular mechanisms remain to be elucidated.

\section{Methods}

The animals were treated in accordance with the guidelines published by the NIH (Guide for the Care and Use of Laboratory Animals), and all experimental procedures were approved prior to study initiation by the Ethics Committee on Experimental Animal Research (Institute of Biological Sciences, Federal University of Pará, Brazil).

\section{Animals and experimental groups}

In this study, 66 mice were housed in groups of 6 in standard plastic cages $(32 \times 39 \times 16.5 \mathrm{~cm})$ covered by metal grids until they were sacrificed. All behavioural assays were performed using 3-, 6- and 18-month old mice. The reduced mastication condition began on the 21 st postnatal day by the introduction of a powder diet (SD mice); control mice (HD mice) were fed a pellet diet. Mice had free access to food and water and were raised under controlled room temperature $\left(23 \pm 1^{\circ} \mathrm{C}\right)$ and 12-h light-dark cycles. There were no significant differences in body weight in the SD and HD experimental groups (measured the day of sacrifice), suggesting that the nutritional value of SD and HD was the same.

Compared to the groups of young mice (3- and 6month-old mice), aging was associated with significant number of deaths. Although we have not found any significant differences in body weight or behaviour before deaths there was greater mortality in the 18-month-old SD group compared to the 18-month-old HD group.

\section{Behavioural tests}

All tests were recorded with a webcam, and images were analysed with a computer program to score mouse performance in open field and water maze tests (ANY-maze Tracking System, Stöelting). Computer analysis was performed off-line. Behavioural tests were performed during the light cycle (08 h-12 h).

\section{Water maze test}

3-month-old (HD3M, n = 12; SD3M, n = 12), 6-monthold (HD6M, n = 12; SD6M, n = 12) and 18-month-old (HD18M, $\mathrm{n}=13$; SD18M, $\mathrm{n}=7$ ) mice were trained in a water maze adapted for mouse dimensions. The circular pool and platform were 94 and $14 \mathrm{~cm}$ in diameter, respectively, and the platform was $1 \mathrm{~cm}$ below the water surface. To hide the black platform, the pool was filled with black water $\left(22 \pm 2^{\circ} \mathrm{C}\right)$ coloured with a non-toxic dye. In each trial, the mice were allowed $60 \mathrm{~s}$ to find the hidden platform; trials were separated by intervals of $30 \mathrm{~s}$. The task was considered complete when animals found and remained on the platform for $10 \mathrm{~s}$. The first day of water maze training allowed the animals to adapt to the aquatic labyrinth. On the remaining four days, animals were tested in three trials once per day. We recorded the escape latency, distance travelled, average swimming speed and trajectories for each mouse. The visual cues outside the water maze were stable on all training days. The learning rate for the water maze was calculated by comparing results on the $1^{\text {st }}$ and $5^{\text {th }}$ test days. All groups were compared using one-way ANOVA, the Bonferroni a priori test or two-way ANOVA followed by Bonferroni post-tests, with intra- or between-group differences considered significant at a $95 \%$ confidence level $(p<0.05)$.

\section{Perfusion and histology procedures}

After the 5-day water maze behavioural tests, the mice were weighed and sacrificed with an overdose of ketamine $(100 \mathrm{mg} / \mathrm{kg}$ ) and xylazine $(10 \mathrm{mg} / \mathrm{kg}$ ) (Konig Laboratories). They were then perfused transcardially with heparinized saline for $10 \mathrm{~min}$, followed by an aldehyde fixative (4\% paraformaldehyde in $0.1 \mathrm{M}$ phosphate buffer, $\mathrm{pH}$ 7.2-7.4) for $30 \mathrm{~min}$. All chemicals were purchased from Sigma (São Paulo, Brazil). After perfusion and craniotomy, the brains were removed and cut on a vibratome at a $70-\mu \mathrm{m}$ thickness. One of each 5 sections was used for GFAP 
detection using free-floating immunohistochemistry. Freefloating sections were rinsed once in $0.1 \mathrm{M}$ phosphate buffer, transferred to $0.2 \mathrm{M}$ boric acid $\mathrm{pH} 9.0$, heated to 65 $70^{\circ} \mathrm{C}$ for $1 \mathrm{~h}$ and then washed $3 \times 5 \mathrm{~min}$ in PBST (5\%). The sections were incubated in a $1 \%$ hydrogen peroxide solution in methanol under constant and gentle shaking for $10 \mathrm{~min}$ and rinsed $2 \times 2 \mathrm{~min}$ in $0.1 \mathrm{M}$ PBS. The sections were then blocked with immunoglobulin for $1 \mathrm{~h}$, following the instructions for the Mouse-on-Mouse Immunodetection kit (M.O.M. kit, Vector Laboratories, USA). Blocking was followed by washing $(3 \times 2 \mathrm{~min})$ in PBS. Sections were incubated in a working solution of protein concentrate for $5 \mathrm{~min}$, then incubated with monoclonal mouse anti-GFAP primary antibody (MAB360, Chemicon International, USA) diluted 1:800 in protein concentrate solution (M.O.M. kit) at $4^{\circ} \mathrm{C}$ for 3 days with continuous gentle agitation. Next, the sections were washed $(3 \times 2 \mathrm{~min})$ in PBS and incubated for $20 \mathrm{~h}$ with biotinylated horse anti-mouse secondary antibody (M.O. M. kit), diluted 1:100 in PBS. After washing $(3 \times 2 \mathrm{~min})$ in $\mathrm{PBS}$, sections were transferred to an avidin-biotin-peroxidase complex solution (ABC, Vector Laboratories, USA, 1:200) for $1.5 \mathrm{~h}$, washed $(3 \times 2 \mathrm{~min})$ in $0.1 \mathrm{M} \mathrm{PB}$, and processed using the glucose oxidase-DAB-nickel method and peroxidase histochemistry (Shu et al. 1988).

The reaction was interrupted when fine astrocytic branches were detected under the microscope. Sections were rinsed $(4 \times 5 \mathrm{~min})$ in $0.1 \mathrm{M} \mathrm{PBS}$, mounted on gelatinized slides, dehydrated in alcohol and xylene, and topped by a coverslip with Enthelan (Merck).

\section{Photomicrograph documentation and processing}

Digital photomicrographs were taken with a digital camera (Microfire, Optronics, CA, USA) coupled to a Nikon microscope (Optiphot-2, NY, USA). The digital photomicrographs were processed with Adobe Photoshop 7.0.1 C. S.2 software (San Jose, CA, USA) for scaling; adjustments to the levels of brightness and contrast were applied to the entire image. The selected micrographs display representative sections from each experimental group i.e. the astrocyte number in each region of interest was close to the mean value for that region.

\section{Microscopy and optical fractionator}

Details of the optical fractionator methodology and experimental parameters are described in online supplementary material (Additional file 5: Table S5, Additional file 6: Table S6, Additional file 7: Table S7 and Additional file 8: Table S8). In brief, we delineated the region and layers of CA1 at all levels in the histological sections, digitizing directly from sections using a low power $3.2 \times$ objective on a Optiphot-2 microscope (Nikon, Japan) equipped with a motorized stage (MAC200, Ludl Electronic Products, Hawthorne, NY, USA). This system was coupled to a computer running Stereoinvestigator software (MicroBrightField, Williston, VT, USA), which was used to store and analyse the $\mathrm{x}, \mathrm{y}$ and $\mathrm{z}$ coordinates of digitized points. To detect and count unambiguously the objects of interest in the dissector probe, the low power objective was replaced by a $60 \times$ oil immersion Plan apochromatic objective (NIKON, NA 1.4) to count astrocytes.

\section{Statistical analysis}

All groups of animals were tested for statistical normality. Possible outliers identified based on standard deviations were eliminated from the data set. Parametric statistical analysis was used to assess the level of significance of the results of behavioural tests, one-way ANOVA, and the Bonferroni a priori test. The significance level for statistical differences was set at alpha < 0.05 (i.e. at a 95\% confidence level). Results from the optical fractionator were analysed using two-way ANOVA. A two-tailed $t$-test was used to compare agematched groups with different diets (SD vs. HD). Statistical analyses were performed using BioEstat ${ }^{\circledR}$ 5.0, Statistica for Windows ${ }^{\circledR}$ version 5.0 A and GraphPad Prism 5 for Windows ${ }^{\circledR}$.

\section{Additional material}

Addtional file 1: Table S1. Estimated Unilateral Number of Astrocytes (N) With the Coefficient of Error (CE) for the Stratum LacunosumMoleculare of CA1 of 3-, 6- and 18-Month-Old Female Albino Swiss Mice Fed A Hard Diet (HD) or Soft Diet (SD).

Additional file 2: Table S2. Estimated Unilateral Number of Astrocytes (N) With the Coefficient of Error (CE) for the Stratum Radiatum of CA1 of 3-, 6- and 18-Month-Old Female Albino Swiss Mice Fed A Hard Diet (HD) or Soft Diet (SD)

Additional file 3: Table S3. Estimated Unilateral Number of Astrocytes (N) With the Coefficient of Error (CE) for the Stratum Pyramidale of CA1 of 3-, 6- and 18-Month-Old Female Albino Swiss Mice Fed A Hard Diet (HD) or Soft Diet (SD).

Additional file 4: Table S4. Estimated Unilateral Number of Astrocytes (N) With the Coefficient of Error (CE) for the Stratum Oriens of CA1 of 36- and 18-Month-Old Female Albino Swiss Mice Fed A Hard Diet (HD) or Soft Diet (SD).

Additional file 5: Table S5. Experimental parameters and optical fractionator counting results in the stratum lacunosum-moleculare of the CA1 of 3-, 6- and 18-month-old female albino swiss mice fed a hard diet (HD) or soft diet (SD).

Additional file 6: Table S6. Experimental Parameters and Optical Fractionator Counting Results in the Stratum Radiatum of CA1 of 3-, 6and 18-Month-Old Female Albino Swiss Mice Fed A Hard Diet (HD) or Soft Diet (SD).

Additional file 7: Table S7. Experimental Parameters and Optical Fractionator Counting Results in the Stratum Pyramidale of CA1 of 3-, 6and 18-Month-Old Female Albino Swiss Mice Fed A Hard Diet (HD) or Soft Diet (SD).

Additional file 8: Table S8. Experimental Parameters and Optical Fractionator Counting Results in the Stratum Oriens of CA1 of 3-, 6- and 18-Month-Old Female Albino Swiss Mice Fed A Hard Diet (HD) or Soft Diet (SD). 


\section{Acknowledgements}

This project was supported by research funds from the Brazilian Government. MCKS was supported by a grant from the Brazilian Research Council CNPq (grant number 475677/2008-0) and from FAPESPA - Fundação de Amparo à Pesquisa do Estado do Pará (grant number 136/08). Research funds from Fundação de Amparo e Desenvolvimento da Pesquisa - FADESP and Pró-Reitoria de Pesquisa e Pós-Graduação - PROPESP/UFPA covered proofreading, edition and publication fees.

\section{Author details}

'Universidade Federal do Pará-UFPA, Instituto de Ciências Biológicas, Laboratório de Investigações em Neurodegeneração e Infecção, Hospital Universitário João de Barros Barreto, Belém, PA, Brazil. ${ }^{2}$ Departamento de Arbovirologia e Febres Hemorrágicas, Instituto Evandro Chagas, IEC, Ananindeua, PA, Brazil. ${ }^{3}$ Southampton Neuroscience Group, School of Biological Sciences, University of Southampton, Southampton SO16 7PX, UK. ${ }^{4}$ Laboratório de Investigações em Neurodegeneração e Infecção, ICB/HUJBB/ UFPA, Rua dos Mundurucus, 4487, Guamá, Belém, PA CEP:66073-000, Brazil.

\section{Authors' contributions}

MNFA, FCCSM, APGF, MF, MLFA, CWPD and MCKS conceived the study, participated in the experiment design and drafted the manuscript. MNFA, FCCSM, APGF, MF, MLFA, CWPD and MCKS performed the experiments and analysed and interpreted the data. JBT, PFCV, VHP, CWPD and MCKS participated in the data analysis, were involved in drafting the manuscript, and made important intellectual contributions. All authors read and approved the final manuscript.

\section{Competing interests}

The authors declare that they have no competing interests.

Received: 4 October 2011 Accepted: 29 February 2012

Published: 29 February 2012

\section{References}

1. Tucha $L$, Simpson $W$ : The role of time on task performance in modifying the effects of gum chewing on attention. Appetite 2011, 56(2):299-301.

2. Tucha L, Simpson W, Evans L, Birrel L, Sontag TA, Lange KW, Tucha O: Detrimental effects of gum chewing on vigilance in children with attention deficit hyperactivity disorder. Appetite 2010, 55(3):679-684.

3. Sakamoto K, Nakata H, Kakigi R: The effect of mastication on human cognitive processing: a study using event-related potentials. Clin Neurophysiol 2009, 120(1):41-50.

4. Weijenberg RA, Scherder EJ, Lobbezoo F: Mastication for the mind-The relationship between mastication and cognition in ageing and dementia. Neurosci Biobehav Rev 2011, 35(3):483-497.

5. Kato T, Usami T, Noda Y, Hasegawa M, Ueda M, Nabeshima T: The effect of the loss of molar teeth on spatial memory and acetylcholine release from the parietal cortex in aged rats. Behav Brain Res 1997, 83(12):239-242.

6. Yamamoto T, Hirayama A: Effects of soft-diet feeding on synaptic density in the hippocampus and parietal cortex of senescence-accelerated mice. Brain Res 2001, 902(2):255-263.

7. Kubo KY, Iwaku F, Watanabe K, Fujita M, Onozuka M: Molarless-induced changes of spines in hippocampal region of SAMP8 mice. Brain Res 2005, 1057(1-2):191-195.

8. Onozuka M, Watanabe K, Nagasaki S, Jiang Y, Ozono S, Nishiyama K, Kawase T, Karasawa N, Nagatsu I: Impairment of spatial memory and changes in astroglial responsiveness following loss of molar teeth in aged SAMP8 mice. Behav Brain Res 2000, 108(2):145-155.

9. Arakawa $Y$, Ichihashi $Y$, linuma $M$, Tamura $Y$, Iwaku F, Kubo KY: Durationdependent effects of the bite-raised condition on hippocampal function in SAMP8 mice. Okajimas folia anatomica Japonica 2007, 84(3):115-119.

10. Kubo KY, Saitoh N, Kogaya Y, Iwaku F, Ichihashi Y, Arakawa Y, Kurata C, linuma $M$, Tamura $Y$ : The bite-raised condition enhances the aging process in the dorsal and ventral hippocampus. Okajimas folia anatomica Japonica 2008, 85(2):43-48.

11. Onozuka M, Watanabe K, Mirbod SM, Ozono S, Nishiyama K, Karasawa N, Nagatsu I: Reduced mastication stimulates impairment of spatial memory and degeneration of hippocampal neurons in aged SAMP8 mice. Brain Res 1999, 826(1):148-153.
12. Kubo KY, Yamada Y, linuma M, Iwaku F, Tamura Y, Watanabe K, Nakamura $\mathrm{H}$, Onozuka M: Occlusal disharmony induces spatial memory impairment and hippocampal neuron degeneration via stress in SAMP8 mice. Neurosci Lett 2007, 414(2):188-191.

13. Watanabe K, Ozono S, Nishiyama K, Saito S, Tonosaki K, Fujita M, Onozuka M: The molarless condition in aged SAMP8 mice attenuates hippocampal Fos induction linked to water maze performance. Behav Brain Res 2002, 128(1):19-25.

14. Aoki H, Kimoto K, Hori N, Hoshi N, Yamamoto T, Onozuka M: Molarless condition suppresses proliferation but not differentiation rates into neurons in the rat dentate gyrus. Neurosci Lett 2010, 469(1):44-48.

15. Watanabe K, Tonosaki K, Kawase T, Karasawa N, Nagatsu I, Fujita M, Onozuka M: Evidence for involvement of dysfunctional teeth in the senile process in the hippocampus of SAMP8 mice. Experimental gerontology 2001, 36(2):283-295.

16. Yamamoto T, Hirayama A, Hosoe N, Furube M, Hirano S: Effects of soft-diet feeding on BDNF expression in hippocampus of mice. The Bulletin of Tokyo Dental College 2008, 49(4):185-190.

17. Yamamoto T, Hirayama A, Hosoe N, Furube M, Hirano S: Soft-diet feeding inhibits adult neurogenesis in hippocampus of mice. The Bulletin of Tokyo Dental College 2009, 50(3):117-124.

18. Tsutsui K, Kaku M, Motokawa M, Tohma Y, Kawata T, Fujita T, Kohno S, Ohtani J, Tenjoh K, Nakano M, et al: Influences of reduced masticatory sensory input from soft-diet feeding upon spatial memory/learning ability in mice. Biomedical research (Tokyo, Japan) 2007, 28(1):1-7.

19. Sesay M, Tanaka A, Ueno Y, Lecaroz P, De Beaufort DG: Assessment of regional cerebral blood flow by xenon-enhanced computed tomography during mastication in humans. Keio J Med 2000, 49(Suppl 1):A125-128.

20. Onozuka M, Fujita M, Watanabe K, Hirano Y, Niwa M, Nishiyama K, Saito S: Mapping brain region activity during chewing: a functional magnetic resonance imaging study. J Dental Res 2002, 81(11):743-746.

21. Hasegawa Y, Ono T, Sakagami J, Hori K, Maeda Y, Hamasaki T, Nokubi T: Influence of voluntary control of masticatory side and rhythm on cerebral hemodynamics. Clin Oral Investig 2011, 15(1):113-118.

22. Figley CR, Stroman PW: The role(s) of astrocytes and astrocyte activity in neurometabolism, neurovascular coupling, and the production of functional neuroimaging signals. Eur J Neurosci 2011, 33(4):577-588.

23. Lecrux $C$, Toussay $X$, Kocharyan A, Fernandes $P$, Neupane $S$, Levesque $M$, Plaisier F, Shmuel A, Cauli B, Hamel E: Pyramidal neurons are "neurogenic hubs" in the neurovascular coupling response to whisker stimulation. J Neurosci 2011, 31(27):9836-9847.

24. Kuga N, Sasaki T, Takahara Y, Matsuki N, Ikegaya Y: Large-scale calcium waves traveling through astrocytic networks in vivo. J Neurosci 2011, 31(7):2607-2614

25. Girouard H, Bonev AD, Hannah RM, Meredith A, Aldrich RW, Nelson MT: Astrocytic endfoot $\mathrm{Ca} 2+$ and BK channels determine both arteriolar dilation and constriction. Proc Natl Acad Sci USA 2010, 107(8):3811-3816.

26. Diniz D, Foro C, Bento-Torres J, Vasconcelos P, CW P-D: Aging, environmental enrichment, object recognition and astrocyte plasticity in dentate gyrus. Astrocytes: Structure, Functions and Role in Disease New York: Nova Science Publisher Inc; 2011.

27. Jinno S: Regional and laminar differences in antigen profiles and spatial distributions of astrocytes in the mouse hippocampus, with reference to aging. Neuroscience 2011, 180:41-52.

28. Morris R: Developments of a water-maze procedure for studying spatial learning in the rat. J Neurosci Methods 1984, 11(1):47-60.

29. Koopmans G, Blokland A, van Nieuwenhuijzen P, Prickaerts J: Assessment of spatial learning abilities of mice in a new circular maze. Physiol Behav 2003, 79(4-5):683-693.

30. Prediger RD, Franco JL, Pandolfo P, Medeiros R, Duarte FS, Di Giunta G, Figueiredo CP, Farina M, Calixto JB, Takahashi RN, et al: Differential susceptibility following beta-amyloid peptide-(1-40) administration in C57BL/6 and Swiss albino mice: Evidence for a dissociation between cognitive deficits and the glutathione system response. Behav Brain Res 2007, 177(2):205-213.

31. Rao SB, Chetana M, Uma Devi P: Centella asiatica treatment during postnatal period enhances learning and memory in mice. Physiol Behav 2005, 86(4):449-457.

32. Diogenes MJ, Costenla AR, Lopes LV, Jeronimo-Santos A, Sousa VC, Fontinha BM, Ribeiro JA, Sebastiao AM: Enhancement of LTP in aged rats 
is dependent on endogenous BDNF. Neuropsychopharmacology 2011, 36(9):1823-1836

33. Bejot Y, Prigent-Tessier A, Cachia C, Giroud M, Mossiat C, Bertrand N, Garnier $P$, Marie C: Time-dependent contribution of non neuronal cells to BDNF production after ischemic stroke in rats. Neurochem Int 2011, 58(1):102-111.

34. Berry A, Greco A, Giorgio M, Pelicci PG, de Kloet R, Alleva E, Minghetti L, Cirulli F: Deletion of the lifespan determinant p66(Shc) improves performance in a spatial memory task, decreases levels of oxidative stress markers in the hippocampus and increases levels of the neurotrophin BDNF in adult mice. Experimental gerontology 2008, 43(3):200-208

35. Knierim JJ, Lee I, Hargreaves EL: Hippocampal place cells: parallel input streams, subregional processing, and implications for episodic memory. Hippocampus 2006, 16(9):755-764.

36. van Groen T, Miettinen P, Kadish I: The entorhinal cortex of the mouse: organization of the projection to the hippocampal formation. Hippocampus 2003, 13(1):133-149.

37. Steward O: Topographic organization of the projections from the entorhinal area to the hippocampal formation of the rat. J Comp Neurol 1976, 167(3):285-314

38. Wyss JM: An autoradiographic study of the efferent connections of the entorhinal cortex in the rat. J Comp Neurol 1981, 199(4):495-512.

39. Buzsaki G: Polysynaptic long-term potentiation: a physiological role of the perforant path-CA3/CA1 pyramidal cell synapse. Brain Res 1988, 455(1):192-195.

40. Remondes M, Schuman EM: Direct cortical input modulates plasticity and spiking in CA1 pyramidal neurons. Nature 2002, 416(6882):736-740.

41. Ang CW, Carlson GC, Coulter DA: Hippocampal CA1 circuitry dynamically gates direct cortical inputs preferentially at theta frequencies. J Neurosci 2005, 25(42):9567-9580

42. Honsek SD, Walz C, Kafitz KW, Rose CR: Astrocyte calcium signals at Schaffer collateral to CA1 pyramidal cell synapses correlate with the number of activated synapses but not with synaptic strength. Hippocampus 2012, 22(1):29-42.

43. Ben Menachem-Zidon O, Avital A, Ben-Menahem $Y$, Goshen I, Kreisel T, Shmueli EM, Segal M, Ben Hur T, Yirmiya R: Astrocytes support hippocampal-dependent memory and long-term potentiation via interleukin-1 signaling. Brain Behav Immun 2011, 25(5):1008-1016.

44. Jourdain P, Bergersen LH, Bhaukaurally K, Bezzi P, Santello M, Domerca M, Matute C, Tonello F, Gundersen V, Volterra A: Glutamate exocytosis from astrocytes controls synaptic strength. Nature neuroscience 2007, 10(3):331-339.

45. Bonansco C, Couve A, Perea G, Ferradas CA, Roncagliolo M, Fuenzalida M: Glutamate released spontaneously from astrocytes sets the threshold for synaptic plasticity. Eur J Neurosci 2011, 33(8):1483-1492.

46. Landfield PW, Rose G, Sandles L, Wohlstadter TC, Lynch G: Patterns of astroglial hypertrophy and neuronal degeneration in the hippocampus of ages, memory-deficient rats. J Gerontol 1977, 32(1):3-12.

47. Lindsey JD, Landfield PW, Lynch G: Early onset and topographical distribution of hypertrophied astrocytes in hippocampus of aging rats: a quantitative study. J Gerontol 1979, 34(5):661-671.

48. Wu Y, Zhang AQ, Yew DT: Age related changes of various markers of astrocytes in senescence-accelerated mice hippocampus. Neurochem Int 2005, 46(7):565-574.

49. Onozuka M, Watanabe K, Fujita M, Tomida M, Ozono S: Changes in the septohippocampal cholinergic system following removal of molar teeth in the aged SAMP8 mouse. Behav Brain Res 2002, 133(2):197-204.

50. Terasawa H, Hirai T, Ninomiya T, Ikeda Y, Ishijima T, Yajima T, Hamaue N, Nagase $Y$, Kang $Y$, Minami M: Influence of tooth-loss and concomitant masticatory alterations on cholinergic neurons in rats: immunohistochemical and biochemical studies. Neurosci Res 2002, 43(4):373-379.

51. Ono $Y$, Yamamoto $T$, Kubo $K Y$, Onozuka M: Occlusion and brain function: mastication as a prevention of cognitive dysfunction. J Oral Rehabil 2010, 37(8):624-640.

52. Godbout JP, Johnson RW: Age and Neuroinflammation: A Lifetime of Psychoneuroimmune Consequences. Immunol Allergy Clinics North Am 2009, 29(2):321-54

53. Mao L, Wang JQ: Adult neural stem/progenitor cells in neurodegenerative repair. Sheng Li Xue Bao 2003, 55(3):233-244.
54. Lee T, Saruta J, Sasaguri K, Sato S, Tsukinoki K: Allowing animals to bite reverses the effects of immobilization stress on hippocampal neurotrophin expression. Brain Res 2008, 1195:43-49.

55. Bartesaghi R, Gessi T: Activation of perforant path neurons to field CA1 by hippocampal projections. Hippocampus 2003, 13(2):235-249.

56. Reitz C, Brickman AM, Brown TR, Manly J, DeCarli C, Small SA, Mayeux R: Linking hippocampal structure and function to memory performance in an aging population. Archives of neurology 2009, 66(11):1385-1392.

57. Horsburgh K, Reimer MM, Holland P, Chen G, Scullion G, Fowler JH: Axonglial disruption: the link between vascular disease and Alzheimer's disease? Biochemical Society transactions 2011, 39(4):881-885.

58. Kaye EK, Valencia A, Baba N, Spiro A, T Dietrich, Garcia Rl: Tooth loss and periodontal disease predict poor cognitive function in older men. $J$ Am Geriatr Soc 2010, 58(4):713-718.

59. Grammas P: Neurovascular dysfunction, inflammation and endothelial activation: implications for the pathogenesis of Alzheimer's disease. $J$ Neuroinflammation 2011, 8:26-38.

60. Hedden T, Gabrieli JD: Insights into the ageing mind: a view from cognitive neuroscience. Nature reviews 2004, 5(2):87-96.

61. Onozuka M, Watanabe K, Fujita M, Tonosaki K, Saito S: Evidence for involvement of glucocorticoid response in the hippocampal changes in aged molarless SAMP8 mice. Behav Brain Res 2002, 131(1-2):125-129.

62. Kim JJ, Diamond DM: The stressed hippocampus, synaptic plasticity and lost memories. Nature reviews 2002, 3(6):453-462.

63. Hori N, Yuyama N, Tamura K: Biting suppresses stress-induced expression of corticotropin-releasing factor (CRF) in the rat hypothalamus. J Dental Res 2004, 83(2):124-128.

64. Bridges N, Slais K, Sykova E: The effects of chronic corticosterone on hippocampal astrocyte numbers: a comparison of male and female Wistar rats. Acta Neurobiol Exp (Wars) 2008, 68(2):131-138.

65. Lambert KG, Gerecke KM, Quadros PS, Doudera E, Jasnow AM, Kinsley CH: Activity-stress increases density of GFAP-immunoreactive astrocytes in the rat hippocampus. Stress (Amsterdam, Netherlands) 2000, 3(4):275-284.

66. Liu WL, Lee YH, Tsai SY, Hsu CY, Sun YY, Yang LY, Tsai SH, Yang WC: Methylprednisolone inhibits the expression of glial fibrillary acidic protein and chondroitin sulfate proteoglycans in reactivated astrocytes. Glia 2008, 56(13):1390-1400.

67. McEwen BS, Alves SE, Bulloch K, Weiland NG: Ovarian steroids and the brain: implications for cognition and aging. Neurology 1997, 48(5 Suppl 7):S8-15.

68. Woolley CS: Estrogen-mediated structural and functional synaptic plasticity in the female rat hippocampus. Horm Behav 1998, 34(2):140-148

69. Sherwin BB: Can estrogen keep you smart? Evidence from clinical studies. J Psychiatry Neurosci 1999, 24(4):315-321.

70. Markowska AL: Sex dimorphisms in the rate of age-related decline in spatial memory: relevance to alterations in the estrous cycle. J Neurosci 1999, 19(18):8122-8133.

71. Martin DM, Wittert G, Burns NR: Gonadal steroids and visuo-spatial abilities in adult males: implications for generalized age-related cognitive decline. Aging Male 2007, 10(1):17-29.

72. Mouton PR, Long JM, Lei DL, Howard V, Jucker M, Calhoun ME, Ingram DK: Age and gender effects on microglia and astrocyte numbers in brains of mice. Brain Res 2002, 956(1):30-35.

73. Diniz DG, Foro CA, Rego CM, Gloria DA, de Oliveira FR, Paes JM, de Sousa AA, Tokuhashi TP, Trindade LS, Turiel MC, et al: Environmental impoverishment and aging alter object recognition, spatial learning, and dentate gyrus astrocytes. Eur J Neurosci 2010, 32(3):509-519.

74. Glaser EM, Wilson PD: The coefficient of error of optical fractionator population size estimates: a computer simulation comparing three estimators. J Microsc 1998, 192:163.

75. Slomianka L, West M: Estimators of the precision of stereological estimates: an example based on the CA1 pyramidal cell layer of rats. Neuroscience 2005, 136:757-767.

doi:10.1186/1471-2202-13-23

Cite this article as: Frota de Almeida et al:: Spatial memory decline after masticatory deprivation and aging is associated with altered laminar distribution of CA1 astrocytes. BMC Neuroscience 2012 13:23. 